\title{
Limited-Area Atmospheric Modeling Using an Unstructured Mesh
}

\author{
William C. Skamarock, Michael G. Duda, and Soyoung Ha \\ National Center for Atmospheric Research, Boulder, Colorado \\ SANG-HUN PARK \\ Yonsei University, Seoul, South Korea
}

(Manuscript received 30 April 2018, in final form 29 July 2018)

\begin{abstract}
A regional configuration of the atmospheric component of the Model for Prediction Across Scales (MPAS-A) is described and evaluated. It employs horizontally unstructured spherical centroidal Voronoi meshes (nominally hexagonal), and lateral boundary conditions used in rectangular grid regional models are adapted to the MPAS-A Voronoi mesh discretization. Test results using a perfect-model assumption show that the lateral boundary conditions are stable and robust. As found in other regional modeling studies, configurations using larger regional domains generally have smaller solution errors compared to configurations employing smaller regional domains. MPAS-A supports variable-resolution meshes, and when regional domains are expanded to include a coarser outer mesh, the variable-resolution configurations recover most of the error reduction compared to a configuration using uniform high resolution, and at much-reduced cost. The wider relaxation-zone region of the variableresolution mesh also helps reconcile differences near the lateral boundary that evolve between the regional model solution and the driving solution, and the configuration is more stable than one using a uniform high-resolution regional mesh. At convection-permitting resolution, solutions produced using global variable-resolution MPAS-A configurations have smaller solution errors than the regional configurations after about $48 \mathrm{~h}$.
\end{abstract}

\section{Introduction}

Limited-area (regional) atmospheric models continue to be used in research, operational numerical weather prediction (NWP), and regional climate studies. Given the success of convection-permitting regional nonhydrostatic model forecasts for short-term prediction of convection (Done et al. 2004) and the very high cost of convection-permitting global simulations, regional simulation capabilities should continue to be employed as a tool in atmospheric research and operational forecasting for the foreseeable future. Regional models, however, bring with them a number of problems associated with their lateral boundary conditions, including questions concerning the well-posedness of the lateral boundary formulations, the potential for solution mismatches between the driving solution and the evolving regional solution, the need for relaxation or sponge zones next to the lateral boundaries, and issues related to the regional domain size and the degree of downscaling employed with a given configuration. Recent papers by Davies

Corresponding author: William C. Skamarock, skamaroc@ucar.edu
(2017, 2014) and Hong and Kanamitsu (2014) provide thorough reviews of much of the work performed to date on these problems.

From a theoretical perspective, lateral boundary condition specification for the equations of motion is formally problematic. The conditions are ill-posed for the inviscid hydrostatic equations (Oliger and Sundström 1978), and while they can in principle be specified in a well-posed manner for the inviscid nonhydrostatic system, no regional nonhydrostatic models use such a formulation to our knowledge. The addition of dissipation in both hydrostatic and nonhydrostatic models circumvents the ill-posedness of the inviscid equations by changing the hyperbolic inviscid equations to parabolic viscous equations, and this dissipation may be explicitly specified in the governing equations, or it may be implicit within the solver (e.g., in upwind transport operators). It is contained within most blending-zone formulations.

Even with the resolution of the ill-posedness by adding dissipation to the equations within the blending zone, practical problems still remain (e.g., wave reflection/ refraction because of solution mismatch at boundaries). Many of these additional sources of error are associated 
with the fact that regional models are employed to downscale solutions from global models: they are employed to fill in the finer-scale structures lacking from the coarserscale global solutions, and problems can arise when largerscale features contained in both the driving solution and regional solution diverge. In some applications that can be viewed as an initial value problem where many of the scales in the flow are considered deterministically predictable, such as short-range regional NWP, solution mismatches are tolerated, given the uncertainties associated with error sources in the regional and driving model forecasts. In contrast, regional climate applications are viewed as boundary value problems, where the large-scale solution is given by the driving climate model solution. Here, mismatches in the solutions are more problematic given the lack of deterministic predictability, and this has led to the use of techniques such as spectral nudging to keep the large scales in the regional and global solutions together. The issues associated these different approaches to downscaling are discussed by Hong and Kanamitsu (2014) and Castro et al. (2005).

The use of unstructured horizontal meshes in global atmospheric models opens the possibility of employing the variable-resolution capabilities of these models to address some of the boundary condition problems arising in limited-area applications. The atmospheric component of the Model for Prediction Across Scales (MPAS-A) allowed only global configurations previous to this study. Herein, we use global and newly developed regional configurations of MPAS-A to examine the benefits of using variable resolution in regional forecasts in addition to validating the implementation of the regional formulation. MPAS-A uses an unstructured spherical centroidal Voronoi mesh that allows smoothly varying resolution (or cell spacing) over global or regional domains. We begin in section 2 by describing how regional meshes are constructed for MPAS-A and by outlining the implementation of the regional boundary conditions for the unstructured MPAS-A Voronoi mesh. This is the first regional implementation of a horizontally unstructured mesh atmospheric model to our knowledge. We present results from a 5-day simulation in section 3 using six MPAS-A configurations. The simulations cover a period of active organized convection over the central United States, which is where our regional configurations are focused. A uniform 3-km global mesh simulation is used as truth to measure simulation errors; thus, we are using a perfect-model framework within this study. Both qualitative and quantitative error measures are presented, and boundary-induced anomalies are illustrated. Regional configuration simulation errors are also compared to errors from global configurations. In section 4 , we consider the question of optimization of MPAS-A configurations for forecast applications, and we summarize our results in section 5 .

\section{Regional MPAS configurations}

\section{a. Regional domains}

In regional Eulerian finite-difference and finite-volume atmospheric models [e.g., WRF (Skamarock et al. 2008), COAMPS (Hodur 1997), and ARPS (Xue et al. 2000)], two mechanisms are employed to accommodate the specified solution on the lateral boundaries. First, an outer row or rows of values in the regional domain is specified through spatial and temporal interpolation from the driving solution. This allows for the evaluation of numerical operators that extend beyond a given interior grid cell, and examples of these are advection operators and pressure gradient operators. We call this region the specified region. Second, a relaxation or sponge layer is applied in the region next to the specified region to damp or smooth differences between the model solution and the driving solution. We call this the relaxation region. Many finite-difference and finite-volume models use a staggered grid, where horizontal velocities are located on gridcell edges or vertices (e.g., WRF, COAMPS, and ARPS use C-grid staggering), and here the relaxation and specified edges or vertices need to be defined.

The spherical centroidal Voronoi mesh used in MPAS is depicted in Fig. 1, which shows three cells on the Voronoi mesh along with a cell from its dual triangular mesh. The MPAS Voronoi mesh does not use a global coordinate, and operators needed in the fluid-flow solver are constructed using coordinates local to the cell, edge, or vertex where the operator is defined (see, e.g., Ringler et al. 2010; Skamarock and Gassmann 2011). In contrast, existing regional models employ a single-coordinate system, mapping their regular rectangular computational domain to a region of the sphere using various projections (e.g., stereographic, Lambert). Their grids and operators are defined through the global coordinate, and the regional domain and its relaxation and specified zones are easily defined.

For MPAS, the standard methodologies to define a regional domain are not readily available because there is no global coordinate in which to define a regional domain. Thus, in order to define regional meshes for MPAS, we have developed a capability to extract them from existing MPAS meshes. Figure 2 depicts two meshes extracted using two different methods. Figure 2a depicts a regional mesh extracted from a global mesh where the region is defined as an ellipse (or circle) on the sphere. MPAS mesh cells do not lie exactly on the 


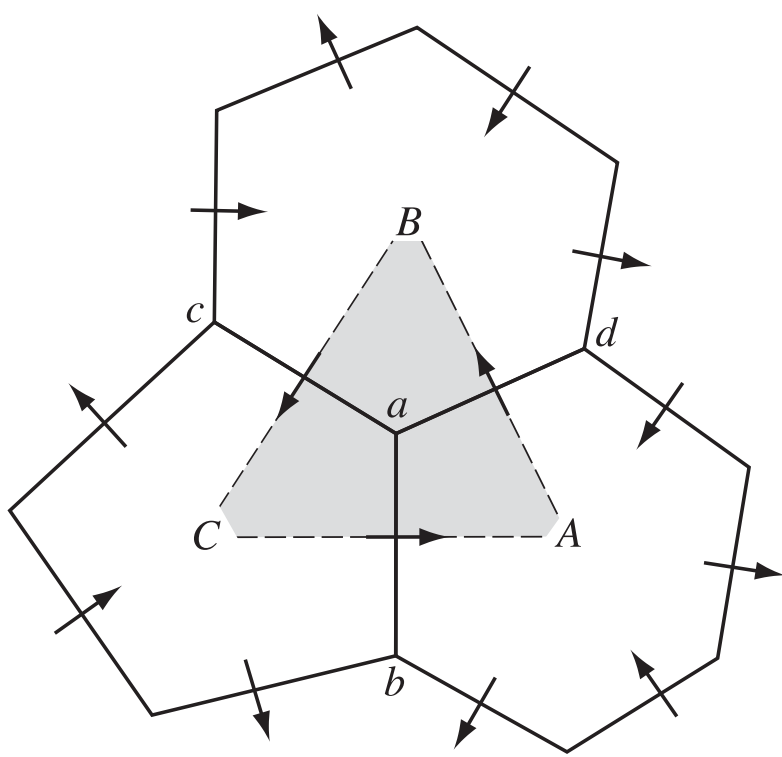

FIG. 1. The C-grid spherical centroidal Voronoi mesh, with Voronoi cell centers $(A, B, C)$, vertices (e.g., $a, b, c, d)$, cell edges (e.g., $a b, a c, a d)$, and normal horizontal prognostic velocities denoted with arrows on cell edges. Cell centers are centroids, the Voronoi property is that cell edges are perpendicular to and bisect lines connecting cell centers (e.g., $a d \perp A B$ ), and all lines are greatcircle arcs on the sphere. The dual triangular mesh cell $A B C$ is shaded. See Ringler et al. (2010) for further details.

ellipse, and the regional-mesh cells are those whose cell centers lie within the ellipse. The boundary relaxation zone and specified zone cells are then added to the mesh by including successive rows of cells exterior to, but neighbors of (sharing an edge with), the interior cells. Figure $2 \mathrm{~b}$ shows a second approach to constructing a regional domain, where the domain is defined by points that are connected by great-circle arcs on the sphere. Paths are constructed by first relocating the points to the nearest Voronoi cell center (the cell that contains a given point) and constructing a path connecting adjoining cell-center points that minimizes the difference between the ideal path (the great-circle arc) and the path made up of cell centers. Interior cells are then identified, and boundary-zone cells are appended.

\section{b. Lateral boundary conditions}

MPAS-A uses a C-grid discretization, as illustrated in Fig. 1, and the lateral boundary conditions must be prescribed for prognostic cell-centered variables and also for the prognostic horizontal normal velocities at the cell edges. Figure 3 shows part of the boundary region of a mesh. We number the levels $1, \ldots, n$, where levels $1, \ldots, m$ correspond to levels in the relaxation region, and levels $m+1, \ldots, n$ are in the specified region. We depict seven levels in the boundary region shown in Fig. 3, with the outer two being the specified region. This is a typical regional MPAS configuration, and it is used to produce all the results presented in this paper. The two specified levels allow the use of the full horizontal flux operators for advection (Skamarock and Gassmann 2011), as well as the full horizontal operators for kinetic energy and vorticity used in the horizontal momentum equation evaluated on the edges (Skamarock et al. 2012; Ringler et al. 2010). The edge levels are
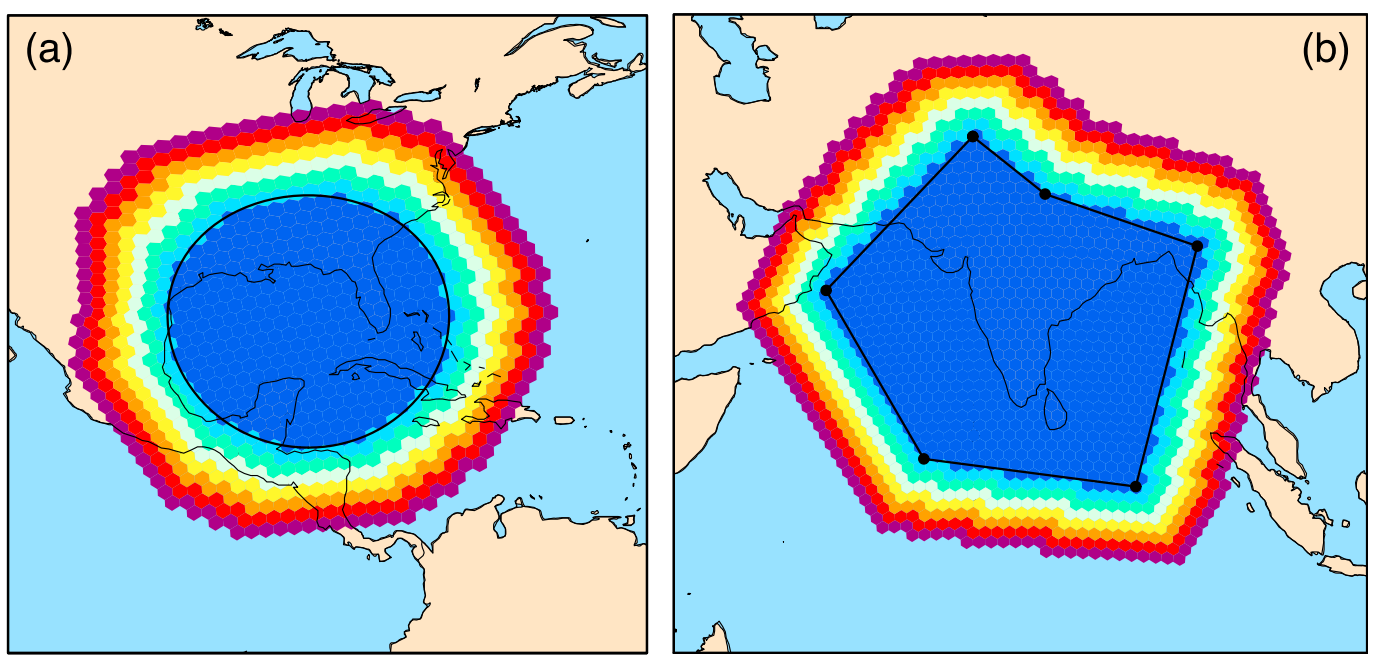

FIG. 2. Regional MPAS meshes extracted from a global MPAS mesh by (a) specifying a circular region and (b) specifying a polygon whose faces are great-circle arcs on the sphere. The regional mesh specifications are given by the black lines, and the blue cells fall in the mesh. The boundary-zone cells appended to the outside of the specified mesh are also shown. 

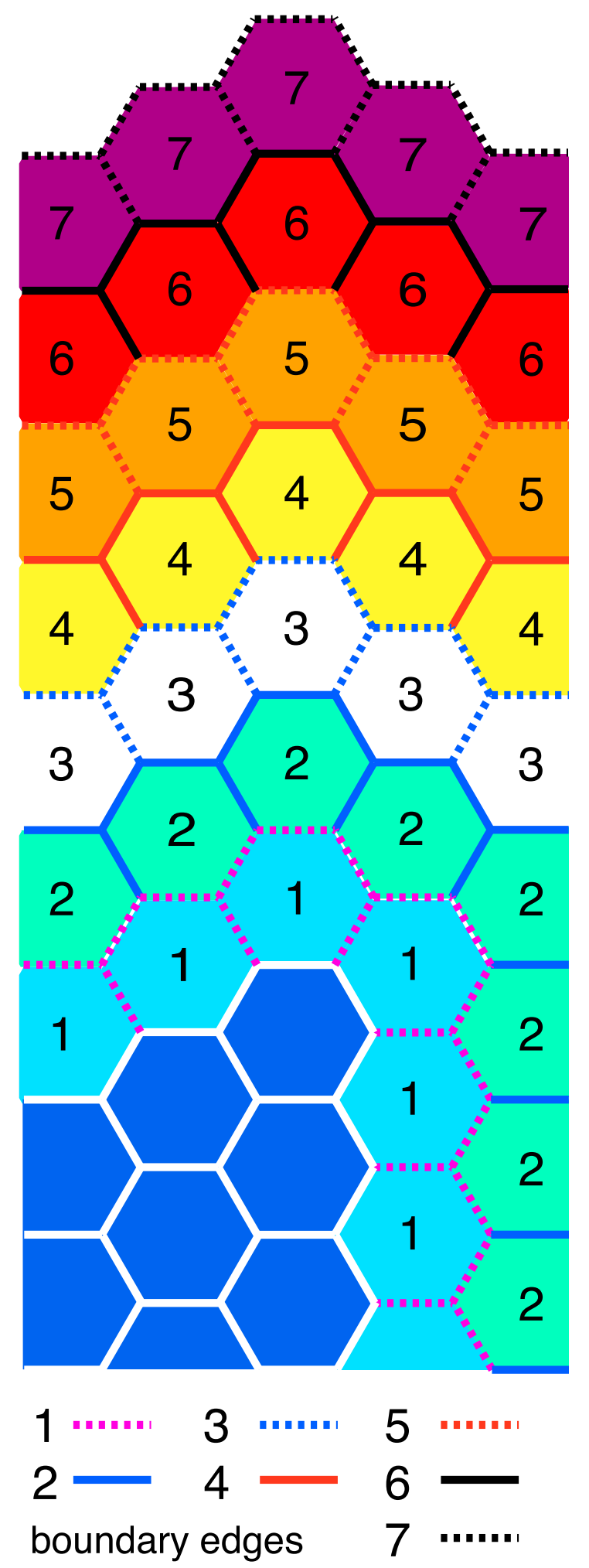

FIG. 3. Boundary zone for the MPAS horizontal mesh. The blue cells are the interior mesh, and the labeled cells and edges are in the boundary region. determined from the cell levels, and the edge level is the smallest level of the two cells sharing that edge.

Within the relaxation region, the MPAS prognostic equations for horizontal and vertical momentum, potential temperature, density, and moisture (vapor and condensates) are augmented by a Rayleigh damping term and a second-order horizontal diffusion of the form

$$
\frac{\partial \psi}{\partial t}=\mathrm{RHS}_{\psi}+F_{1}\left(\psi_{\mathrm{LS}}-\psi\right)-F_{2} \Delta x^{2} \nabla^{2}\left(\psi_{\mathrm{LS}}-\psi\right),
$$

where

$$
F_{1}=\gamma_{1}(i-1) / m, \quad F_{2}=\gamma_{2}(i-1) / m,
$$

for cells $i=2, \ldots, m$ in the relaxation region, $\psi$ is the model state, and $\psi_{\mathrm{LS}}$ is the large-scale driving solution. This formulation follows that described by Davies and Turner (1977), and it is the same as used in the regional WRF lateral boundary conditions (Skamarock et al. 2008), where the solution is damped to the driving solution and the Laplacian operates on the difference between the driving and regional solutions. The MPAS operators for the Laplacian are given in Skamarock et al.'s (2012) Eqs. (15) and (16) for cell-centered variables and horizontal momentum, respectively. It is worth noting that, as is apparent in (2), the values in the first-level cells in the relaxation zone $i=1$ are not altered by the additional operators in (1); this first level is included to fill out the stencil for the rightmost term in (1), the second-order filter.

WRF uses the formulations (1) and (2) with relaxation coefficients $\gamma_{1}=(10 \Delta t)^{-1}$ and $\gamma_{2}=(50 \Delta t)^{-1}$. Both coefficients have units of inverse time $\mathrm{T}^{-1}$. In (1), the term $F_{1}$ is a relaxation time scale, and the term multiplying the Laplacian is a viscosity $F_{2} \Delta x^{2}$ with units $\mathrm{L}^{2} \mathrm{~T}^{-1}$. The relaxation time scale and the implied eddy viscosity scale with the time step in WRF. Moreover, WRF uses a time step that scales with the grid spacing (i.e., WRF applications are typically configured so that $\Delta t \sim 0.006 \Delta x$ ). Thus, the relaxation time scale and eddy viscosity scale with the grid spacing and time step in WRF. MPAS, however, can have cell spacing that varies substantially over the mesh, while the time step is limited by the finest cell spacing on the mesh. Thus, if we set the coefficients $F_{1}$ and $F_{2}$ based on the time step, they would have a relaxation time scale and viscosities that would be too small in the coarser regions of the mesh. To circumvent this problem, we specify the boundary relaxation and filtering coefficients such that they are independent of the time step in the MPAS configuration, and the relaxation coefficients (2) are specified as $\gamma_{1}=(0.06 \Delta x)^{-1}$ and $\gamma_{2}=(0.3 \Delta x)^{-1}$, where $\Delta x$ is the local cell spacing in the relaxation region. This specification results in the same effective filtering as 
WRF for a given cell spacing. Finally, the relaxation terms involving $\Delta x$ in (1) and in $\gamma_{1}$ and $\gamma_{2}$ are evaluated using the local mesh spacing derived from the density function used to generate the mesh (see Ringler et al. 2011). We use this smooth analytic density function so that the relaxation varies smoothly across the variableresolution mesh, thus minimizing any grid imprinting.

Cell and edge prognostic variables within the specified zone of the regional MPAS mesh are also specified as in WRF. For the case where a global MPAS solution drives a regional MPAS solution, the full state is set in the specified region using spatial and temporal interpolation, including vertical velocity and all moist species. For the case where the driving solution does not contain the full MPAS state (typically lacking vertical velocity and condensates), a zero-gradient condition is applied on outflow for condensates and other unspecified scalars, and fluxes are set to zero on inflow. Within MPAS, these conditions are enabled by setting the specified values to zero and using a first-order upwind (donor cell) flux evaluation for the two outermost sets of edges in the relaxation zone (i.e., edges 4 and 5 in Fig. 3). A zero horizontal gradient condition is used for setting the vertical velocity values in the specified region in WRF, and this condition is approximated in MPAS by setting the specified value of a vertical velocity to that of the nearest cell in the relaxation zone.

MPAS-A uses a horizontally explicit vertically implicit (HEVI) time integration scheme for the acoustic modes as described in Klemp et al. (2007), and the HEVI scheme is used within a Runge-Kutta-based splitexplicit integration technique described in Wicker and Skamarock (2002). The Runge-Kutta integration handles the meteorological (slow) modes, and the relaxation terms in (1) are computed on the Runge-Kutta step. The values in the specified zone are linearly interpolated in time from the driving solution to prescribe the specified state for both the Runge-Kutta step and the acoustic substeps.

Spatial interpolations from MPAS driving simulations to the regional MPAS boundary region employ barycentric interpolation for cell-centered values. The barycentric interpolation uses the dual of the hexagonal mesh, the triangular mesh formed by connecting the cell centers, where the cell-center values are located at the vertices of the triangles (see Fig. 1). To produce the driving solution cell-edge normal velocities in the regional mesh boundary region, the driving solution cell-centered zonal and meridional velocities are interpolated to the cell edges using barycentric interpolation, and the edge-normal component of this vector is recovered. The cell-centered zonal and meridional velocities are computed as part of the MPAS-A solution procedure using radial basis functions (Bonaventura et al. 2011).
The terrain on the regional MPAS mesh is taken from the MPAS mesh from which the regional mesh is extracted. As is the case in many regional modeling systems (WRF; Skamarock et al. 2008), the terrain in the LBC region of the regional mesh is blended with the terrain from the model configuration providing the boundary values. This terrain blending is performed before the extraction process (i.e., the blending occurs on the global mesh in the area where the boundary region will appear on the regional mesh). This procedure also facilitates the coordinate smoothing in the terrain-following formulation used in MPAS-A (Klemp 2011) because it removes the need for any lateral boundary conditions in the smoothing process in the case where a regional mesh is extracted from a global mesh.

For initialization from an MPAS driving solution, the regional configuration of MPAS uses the same initialization procedure as used for the global configuration of MPAS. As a consequence, the regional mesh has exactly the same initial state as would the global mesh from which the regional mesh was extracted, except for boundary regions where terrain blending alters the original vertical mesh. The National Centers for Environmental Prediction (NCEP) Global Forecast System (GFS) Final (FNL) Operational Global Analysis $\left(0.25^{\circ} \times 0.25^{\circ}\right)$ is used to initialize all MPAS regional and global simulations presented in this paper.

\section{Results}

\section{a. Simulation configurations}

Regional models in NWP are used almost exclusively at convection-permitting resolutions, and we have chosen to use a period of strong convection over the central United States as a test case for evaluating regional MPAS configurations. To examine the effects of the regional boundary conditions, we use a uniform $3-\mathrm{km}$ global MPAS simulation as the truth for evaluating the regional configurations of MPAS. We are using a perfect-model approach (de Elía et al. 2002). We could compare directly with observations or other analyses, but this would include sampling model error not associated with the boundary conditions or would be dominated by the representativeness error associated with the coarse resolution of the analysis relative to our simulations. Herein, we are considering short-term weather prediction, with the understanding that the results apply to regional climate applications as well (Hong and Kanamitsu 2014).

Five-day simulations are generated with initializations from the GFS FNL at 0000 UTC 26 April 2017. The mesh configurations used in this study are given in Table 1. Three global simulations are generated using a 
TABLE 1. Characteristics of the horizontal meshes used in the experiments. The cost (processor hours) is normalized by that of the 3-km small regional mesh simulation. The $15-\mathrm{km}$ global simulation cost reflects the 5-times-larger time step used on this mesh, compared to that used in the other simulations.

\begin{tabular}{|c|c|c|c|c|c|}
\hline Mesh No. & Domain/mesh & Use & No. of cells & Area $\left(\mathrm{km}^{2}\right)$ & $\begin{array}{l}\text { Normalized } \\
\text { cost }\end{array}$ \\
\hline \multicolumn{6}{|c|}{ Global meshes } \\
\hline 1 & 3-km global & Reference simulation (truth) & 65536002 & $5.1 \times 10^{8}$ & 305 \\
\hline 2 & $15-\mathrm{km}$ global & $\begin{array}{l}\text { Provides LBC data for regional } \\
\text { simulations }\end{array}$ & 2621442 & $5.1 \times 10^{8}$ & 2.44 \\
\hline 3 & $60-15-3-\mathrm{km}$ global & Global variable resolution reference & 794626 & $5.1 \times 10^{8}$ & 3.70 \\
\hline \multicolumn{6}{|c|}{ Regional meshes } \\
\hline 4 & 3-km large regional & $\begin{array}{l}\text { Large regional mesh (uniform) generated } \\
\text { from mesh } 1\end{array}$ & 1629704 & $1.25 \times 10^{7}$ & 7.60 \\
\hline 5 & 15-3-km regional & $\begin{array}{l}\text { Large regional mesh (variable) generated } \\
\text { from mesh } 3\end{array}$ & 374567 & $1.26 \times 10^{7}$ & 1.75 \\
\hline 6 & 3-km small regional & $\begin{array}{l}\text { Small regional mesh (uniform) generated } \\
\text { from mesh } 3\end{array}$ & 214552 & $1.78 \times 10^{6}$ & 1 \\
\hline
\end{tabular}

uniform 3-km mesh, a uniform 15-km mesh, and a 60-15-3-km variable-resolution mesh. The variableresolution global mesh is depicted in Fig. 4. Three regional simulations are produced using domains centered over the central United States, including a small 3-km domain, a large 3-km domain, and a 15-3-km variableresolution domain that covers the same geographic area as the large 3-km domain. The regional meshes are also depicted in Fig. 4. The 15-3- and 3-km small domains are extracted from the $60-15-3-\mathrm{km}$ global mesh, and the large $3-\mathrm{km}$ regional domain is extracted from the $3-\mathrm{km}$ global mesh. The difference in cell spacing, from 3 to $15 \mathrm{~km}$ (a factor of 5), is larger than used in many regional models or between nests in NWP applications using nested models, but not necessarily large compared to some regional climate configurations.

MPAS is configured with 55 vertical layers and uses a 30-km model top. The level distribution through the troposphere is similar to that used in the GFS forecast configuration. Information about the meshes (e.g., the number of columns in a mesh and the relative simulation cost) is given in Table 1. Boundary values for all regional simulations are spatially and temporally interpolated from the $15-\mathrm{km}$ uniform mesh simulation. Figure 5 shows the 10-m zonal wind and 2-m temperature simulation error, integrated over the region covered by the small 3-km regional domain, for lateral boundary update intervals of $12,6,3$, and $1 \mathrm{~h}$. The errors for the
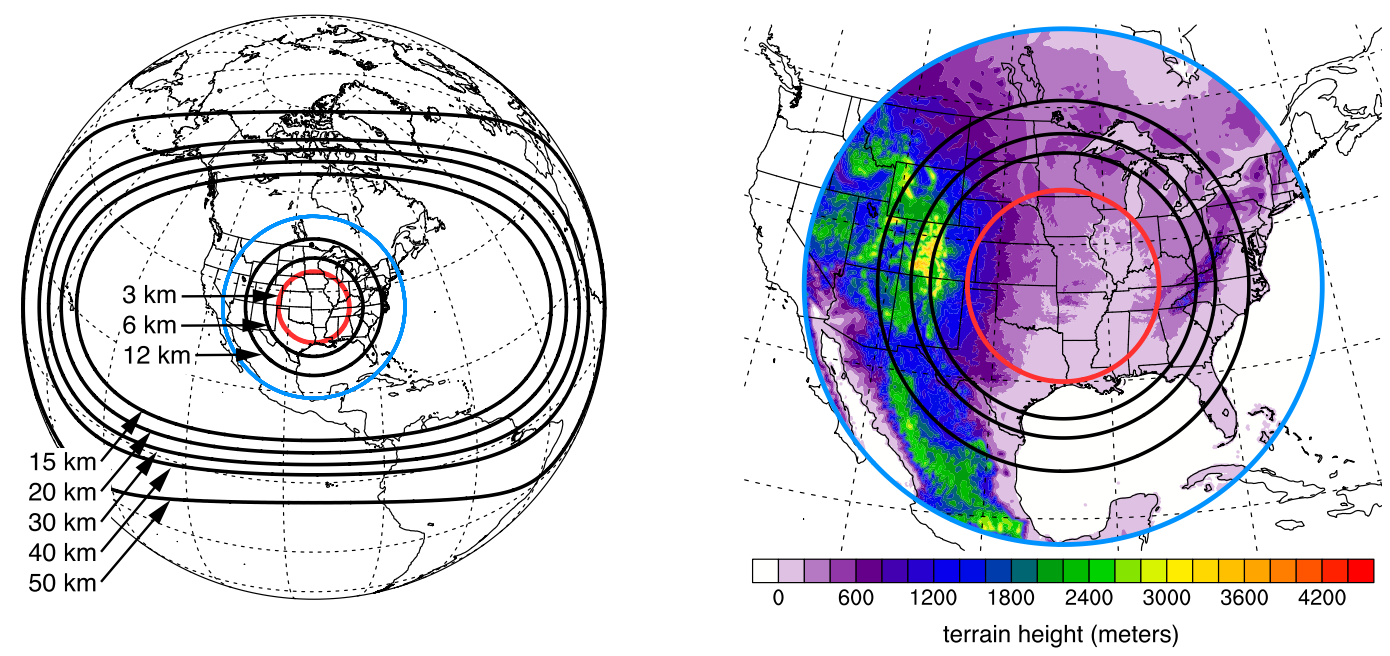

FIG. 4. (left) Global mesh and (right) regional meshes. The cell spacing in the 60-15-3-km variable-resolution global mesh is contoured. The 3-km small regional mesh domain is indicated in red in both plots, the $15-3-\mathrm{km}$ mesh domain is indicated in blue, and the $3-\mathrm{km}$ large domain covers the same (blue) region. The cell spacing is approximately $15 \mathrm{~km}$ in the vicinity of the blue line for the variable-resolution meshes used in these experiments. The cell spacing contour interval in the regional plot is $3 \mathrm{~km}$. 

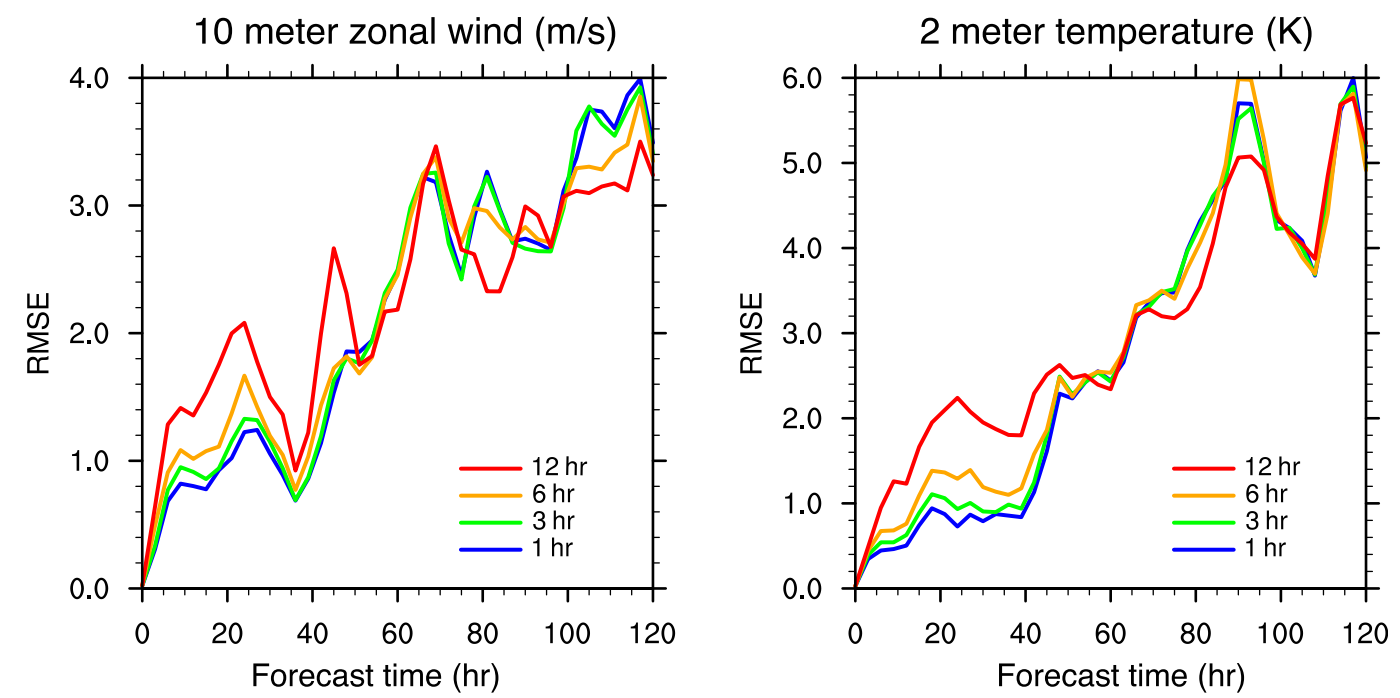

FIG. 5. RMSEs for the 10-m zonal wind and 2-m temperature using different lateral boundary update intervals for the small $3-\mathrm{km}$ regional domain.

3- and 1-hourly update interval closely follow each other over the 5-day simulation, and they are lower than the errors from the 6- and 12-hourly update interval simulations through $48 \mathrm{~h}$, after which the errors are comparable for all intervals. The remainder of the simulation results presented in this paper were generated using a 1-h update interval.

The physics used is the convection_permitting suite used in the MPAS-A version 5.3 release. Importantly, the suite uses the Grell-Freitas scale-aware convection scheme (Grell and Freitas 2014) that has been employed and evaluated in global MPAS-A simulations (Fowler et al. 2016). The scheme models shallow, midlevel, and deep convection. On coarse meshes (cell spacing greater than $20 \mathrm{~km}$ ), the convection scheme handles most of the convective instability, and on fine meshes (less than 6-km cell spacing) it effectively becomes a shallow convection scheme; and there is a smooth transition in behavior between these mesh spacings. Hong and Kanamitsu (2014) discuss the need for scale-aware (or scale insensitive) physics in atmospheric models in the context of regional modeling and downscaling. When using variable-resolution meshes with MPAS-A, the need for scale-aware physics is amplified, given that different resolutions are present on the single mesh, as opposed to the single resolutions differing for a regional model and its driving model or within multinested applications.

\section{b. Regional simulation overview}

Figure 6 depicts the 500-hPa heights and temperature from the $3-\mathrm{km}$ large regional simulation. A large-scale trough is approaching the south-central United States from the west, with a strong upper-level jet persisting over much of the simulation period. Low-level moist southerly flow from the Gulf of Mexico, combined with the strong vertical wind shear, provided favorable conditions for strong convection during this simulation period over the central United States. The first trough propagated through the central United States during 26-27 April, as seen at 0 and $48 \mathrm{~h}$ in Fig. 6, followed by a second deep trough evident at $84 \mathrm{~h}$.

The entire regional solution, including the relaxation zone (five cells) and specified zone (two cells), is plotted in Fig. 6. There are no obvious issues with the lateral boundary conditions evident in the height and temperature fields in these contiguous United States (CONUS)scale plots, even though a strong jet is entering the western region and exiting in the east; the height and temperature fields are smooth up to the boundary in all regions. There is significant structure in the height fields as the jet traverses the Rocky Mountains in the western United States, and the amplification of that structure is expected given that the $3-\mathrm{km}$ regional mesh resolves finer scales in the terrain (at 48 and $84 \mathrm{~h}$ in the figure) compared to that used to produce the $0.25^{\circ} \times 0.25^{\circ} \mathrm{GFS}$ analysis (the initial state at $0 \mathrm{~h}$ in the figure). The boundary region has a width of approximately $21 \mathrm{~km}$, so it would be difficult to discern any boundary issues from a plot at this scale.

The column-maximum reflectivity is plotted in Fig. 7 at simulation hour 24 for the global 3-km reference solution and the small $3-\mathrm{km}$ regional simulation, where the gray lines indicate the outer edge of the $3-\mathrm{km}$ small regional domain in the plots. The observed radar reflectivity is also plotted for reference. The convective system structures are very similar in the two simulations 

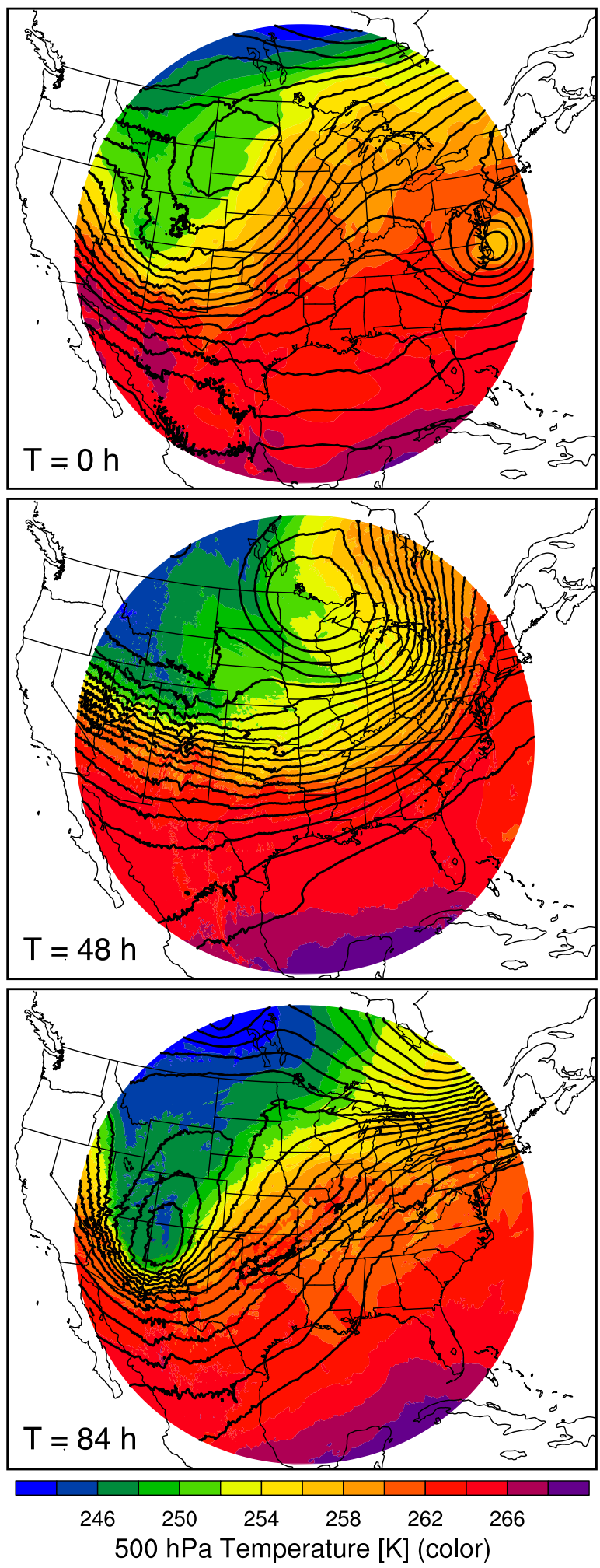

FIG. 6. The 500-hPa height (contour) and temperature fields (fill) from the large $3-\mathrm{km}$ regional simulation at (top) the initial time 0000 UTC 26 Apr 2017 and hours (middle) 48 and (bottom) 84.
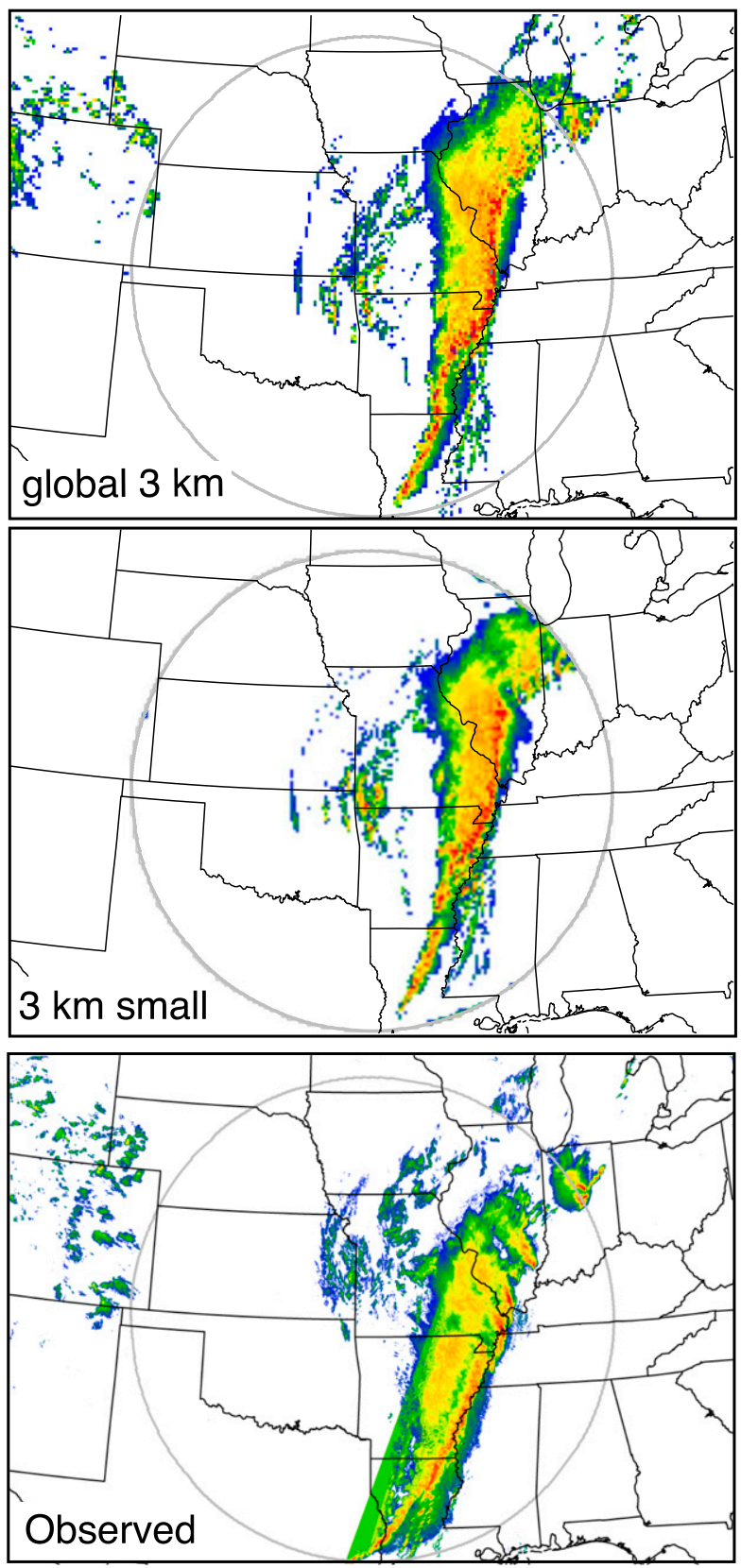

10141822263034384246505458626670

FIG. 7. Simulated column maximum reflectivity (dBZ) at 0000 UTC 27 Apr 2017 (24 h) from the (top) 3-km global simulation and the (middle) 3-km small region, along with the (bottom) observed composite reflectivity from the NOAA MRMS data.

at this time, and both results compare well to the radar observations. The other regional simulation reflectivities (not shown) are also very similar. The reflectivity in the 3-km small regional simulation does not show any signs of boundary condition issues at $24 \mathrm{~h}$. The 48 -h results on all the $3-\mathrm{km}$ meshes also show similar behavior; there are 

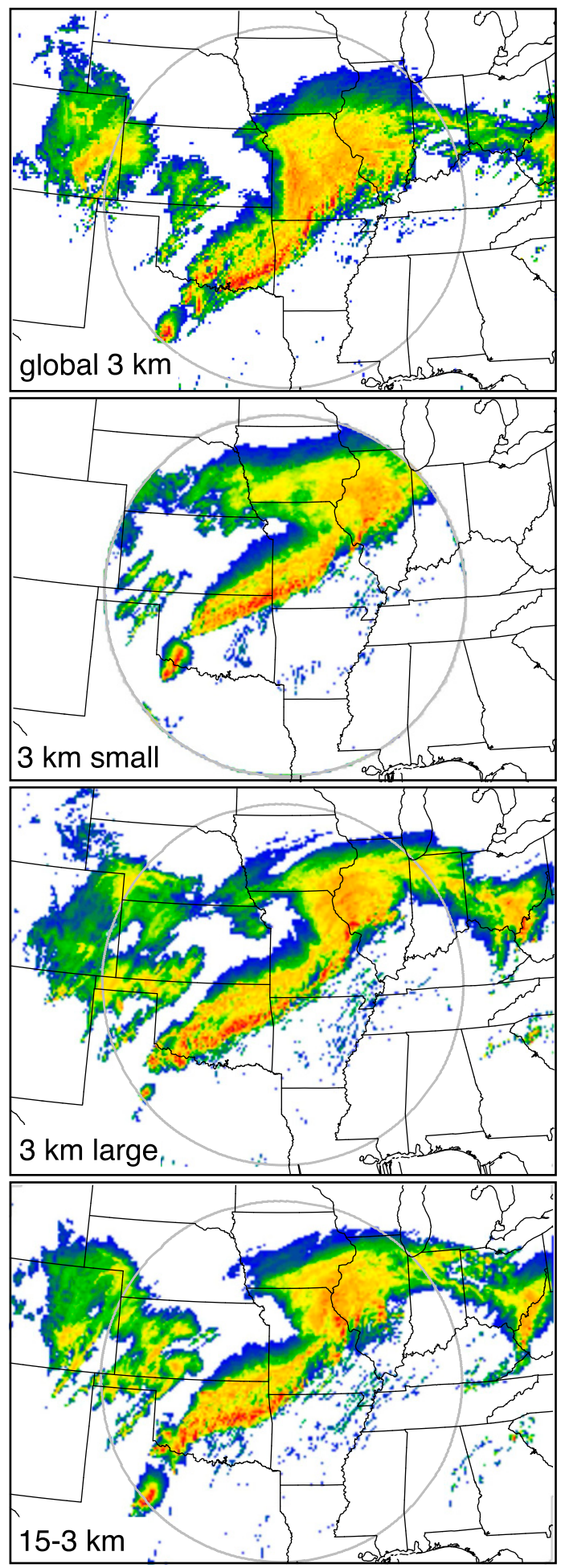

10141822263034384246505458626670

FIG. 8. Column maximum reflectivity (dBZ) from the (top) $3-\mathrm{km}$ global simulation and the three regional simulations at 1200 UTC 29 Apr 2017 (84 h).

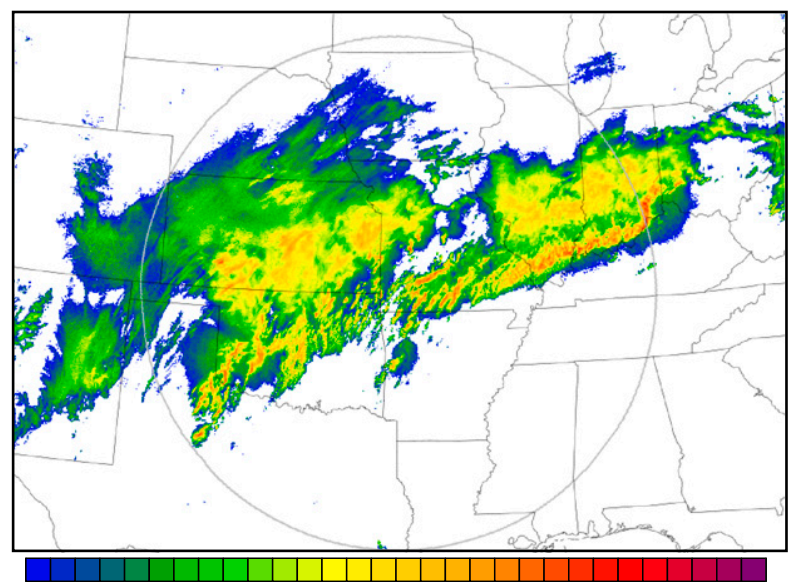

10141822263034384246505458626670

FIG. 9. Observed composite reflectivity (dBZ) at 1200 UTC 29 Apr 2017.

only small differences between the results, and no boundary condition problems are evident even though precipitating structures are propagating out of the $3-\mathrm{km}$ small domain.

The next significant convective outbreak occurs between days 3 and 4 . Figure 8 depicts the column maximum reflectivity at $84 \mathrm{~h}$ for the three regional simulations and for the 3-km global simulation, and Fig. 9 shows the observed radar reflectivity. The convective system structure is broadly similar among the simulations and has some resemblance to the observed system. All the simulations overpredict the leading edge reflectivity and do not capture well the trailing stratiform region over $\mathrm{Ne}$ braska. The 3-km global simulation places the leading edge of the convective system farther to the southeast relative to the regional simulations (see the convective line position in Arkansas, Missouri, and Illinois). The 3-km large and 15-3-km regional simulations also capture the convection in eastern Colorado and in Indiana and Ohio, evident in the 3-km global result and in the observations, although the stratiform regions in the simulations are not as extensive as observed. The 3-km small region simulation is not quickly developing the convection entering its domain from eastern Colorado because of its very weak representation in the global $15-\mathrm{km}$ solution driving this regional solution.

\section{c. Quantitative simulation errors}

Quantitative error measures are computed over the region covered by the small 3-km domain (see Fig. 4) for all simulations using the $3-\mathrm{km}$ global simulation as the truth. Figure 10 shows the root-mean-square error (RMSE) for mean sea level pressure (MSLP) for all five simulations. The $15-\mathrm{km}$ global simulation has the largest 

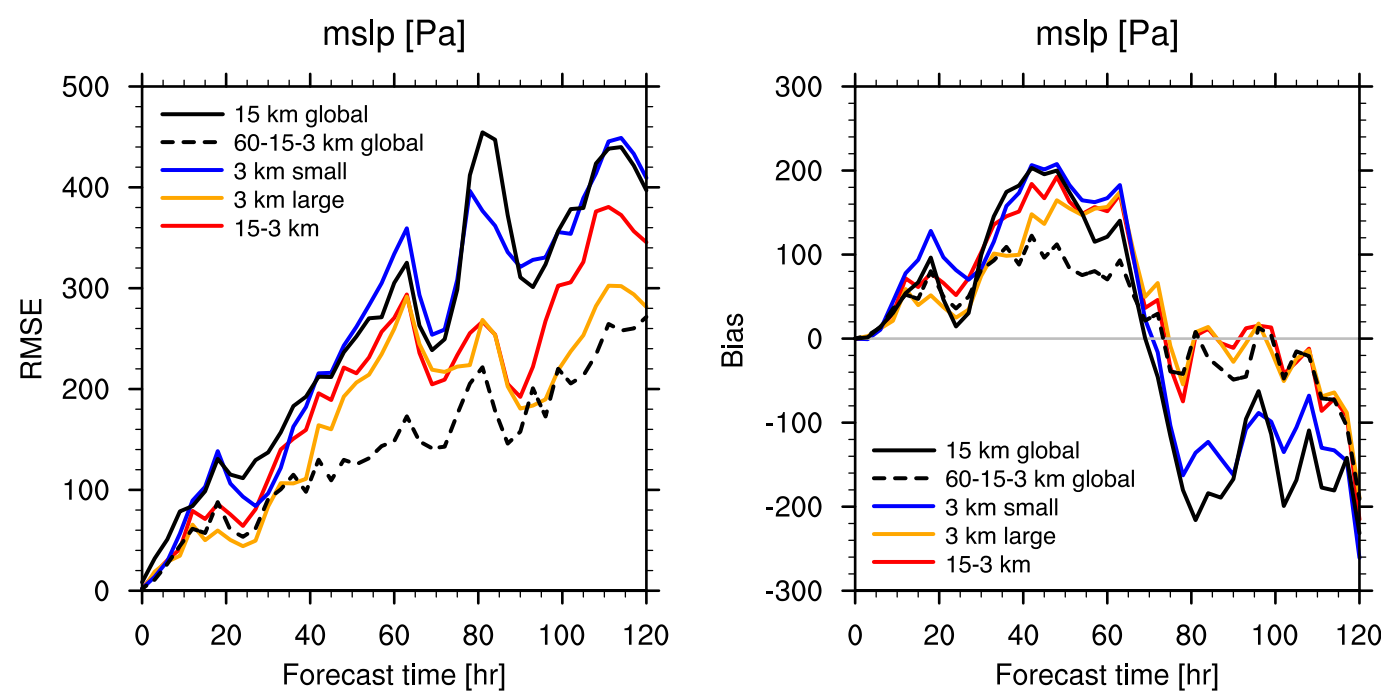

FIG. 10. MSLP RMSE and bias for the simulations computed within the region covered by the small 3-km regional mesh (red circles in Fig. 4).

error through most of the simulation period, although occasionally the $3-\mathrm{km}$ small region simulation has higher error. The 3-km large domain and the $15-3-\mathrm{km}$ regional domain have similar errors through much of the period, with the 15-3-km region simulation typically having a slightly larger error at any given time. The difference gets significantly larger during the last day of the simulation. The MSLP errors for the $15-3-\mathrm{km}$ region and 3-km large region simulations are usually significantly smaller than those of the $15-\mathrm{km}$ global and $3-\mathrm{km}$ small region simulations. The $60-15-3-\mathrm{km}$ simulation has the smallest errors overall, matching those from the $15-3-\mathrm{km}$ forecast and the 3-km large region simulation over the first 1.5 days and then showing a significantly lower error until late in the simulation period. The MSLP biases are also plotted in Fig. 10 and are similar in character to the RMSE, with tightly clustered biases for the $3-\mathrm{km}$ large regional, $15-3-\mathrm{km}$ regional, and $60-15-3-\mathrm{km}$ global simulations. Interestingly, the global $60-15-3-\mathrm{km}$ simulation shows a period of reduced bias between hours 40 and 60 . The $15-\mathrm{km}$ global and 3-km small regional simulations have comparable biases, and while they track the other simulation biases early in the simulation period, they become much larger after 3 days.

The 500-hPa errors plotted as a function of time for the zonal velocity, temperature, and heights are given in Fig. 11. These error metrics are computed over the 3-km small region (see Fig. 4). The zonal velocity errors are similar for all the regional simulations from 40 through $100 \mathrm{~h}$, with larger errors for the $3-\mathrm{km}$ small region and $15-\mathrm{km}$ global simulations outside this period. The 60-15-3-km simulation shows smaller errors than all simulations for the zonal wind after simulation hour 40 , and this characteristic also holds for the temperature and height errors. The temperature and height errors are consistently larger for the $15-$ and $3-\mathrm{km}$ small region simulations relative to the other regional simulations.

The vertical structures of the temperature and zonal wind RMSEs and biases are depicted in Fig. 12. The $15-\mathrm{km}$ global simulation and 3-km small region simulation have the highest errors and biases, the $60-15-3-\mathrm{km}$ global simulation has the smallest errors and biases, and the 3-km large region and $15-3-\mathrm{km}$ regional simulations have errors and biases between those from the other simulations. These errors and biases are similar in structure, but slightly larger at $120 \mathrm{~h}$ (not shown) and similar but reduced at earlier times (also not shown). At all times, the errors in the 3-km small region simulation closely follow those of the $15-\mathrm{km}$ global simulation; the 15-3-km region and 3-km large region simulation errors and biases closely follow each other, and the 60-15-3-km simulations errors and biases are always smaller than the others.

Errors computed over the larger region covered by the 3-km large domain and the $15-3-\mathrm{km}$ domain are plotted for the $500-\mathrm{hPa}$ zonal wind, temperature, and height fields in Fig. 13. These errors are somewhat smaller in magnitude because the larger region is not so tightly focused on the jet, compared to the $3-\mathrm{km}$ small region, but the errors have a similar temporal structure. Specifically, the $15-\mathrm{km}$ global solution has the largest error throughout the simulation period; the $15-3-\mathrm{km}$ regional simulation has very similar and slightly larger errors over most of the simulation period compared to the $3-\mathrm{km}$ large domain; and the $60-15-3-\mathrm{km}$ global simulation has the smallest error overall. 

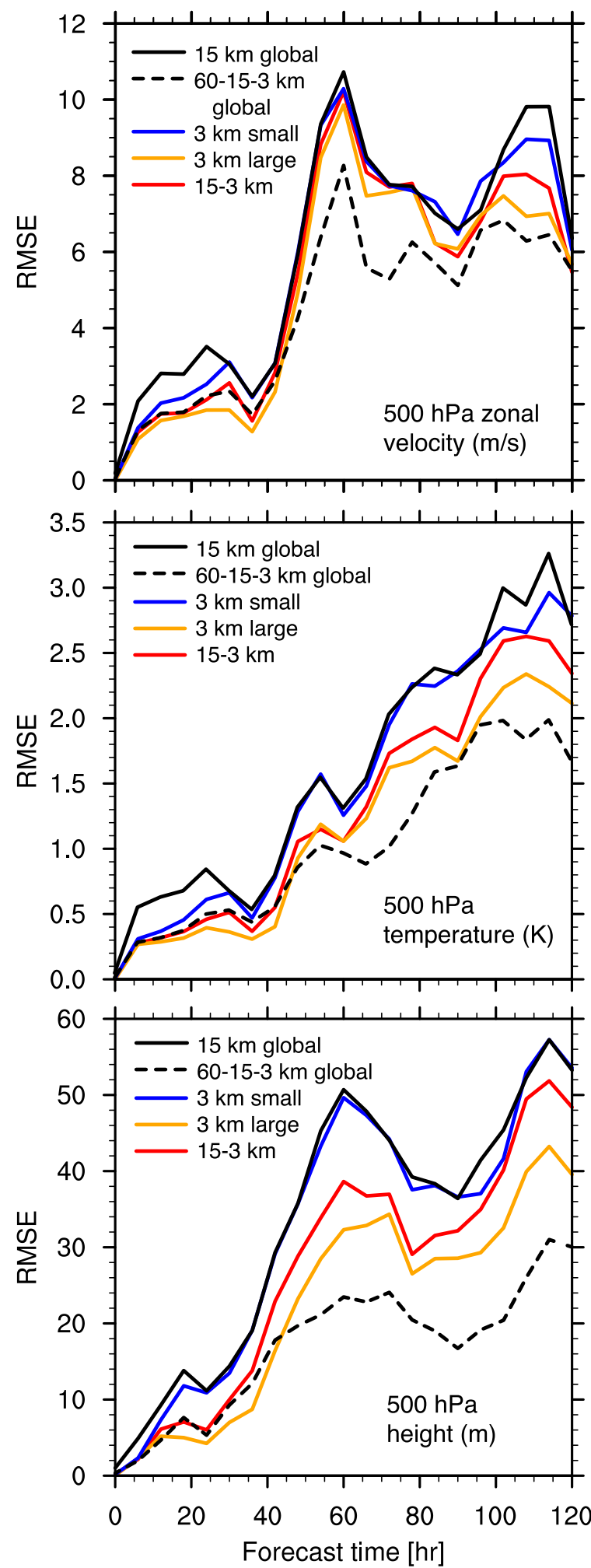

FIG. 11. The 500-hPa wind, temperature, and height errors for the regional simulations computed within the region covered by the small 3-km regional mesh (red circles in Fig. 4).

\section{d. Lateral boundary-induced anomalies}

The 3-km small regional simulation does not show any obvious boundary anomalies. We have observed boundary anomalies in some of the solutions when using larger regional domains, and we have had instances of the integrations becoming unstable because of these anomalies. An example of one such anomaly is given in Fig. 14, which depicts the 500-hPa zonal wind along a portion of the eastern boundaries for the $15-3-\mathrm{km}$ regional simulation and for the 3-km large region simulation. The plots are for simulation hour 84 , and there is a jet exiting the eastern boundary just to the north of the region shown in the figure. The $15-\mathrm{km}$ global simulation provides boundary conditions for the regional simulations, and the jet axis is located slightly north in the $15-\mathrm{km}$ simulation relative to its location in the regional simulations. The mismatch in the zonal velocities results in a ridge in the zonal velocity field at the inner edge of the boundary zone in the regional simulations. This spurious rapid deceleration of the flow over the width of the boundary zone is accompanied by strong convergence and can lead to model instability in some simulations. The boundary-zone width is a fixed number of cells in these MPAS experiments, and this is typical in configurations of other regional models used in research (both NWP and regional climate) and operational NWP. Thus, the spurious convergence and stability problems related to it can be more severe for finer regional meshes. Additionally, mismatched fields in the boundary region between the driving solution and regional model solution become more prevalent for larger regional domains because, compared to smaller domains, the solution features have larger spatial domains and temporal periods over which to evolve and diverge from the driving solution as they propagate through the domain. This is a problem for all regional models, and it is discussed by Hong and Kanamitsu (2014) and Davies $(2014,2017)$ and is illustrated in idealized tests by Park et al. (2014).

There are a number of approaches used to address this solution mismatch problem. Stretched regional grids can be used within rectangular grid models enabling a larger boundary region, as examined in detail by Davies (2017). This approach can also be applied globally (e.g., Côté et al. 1998), although both the regional and global stretching results in highly anisotropic grid cells in the stretched region. Within modeling systems that allow for two-way nesting (e.g., WRF), multiple interacting nested grids can be used to transition from larger outer domains that have large boundary regions because of their coarser outer grids to much smaller inner domains covering the region of interest. The two-way interaction 

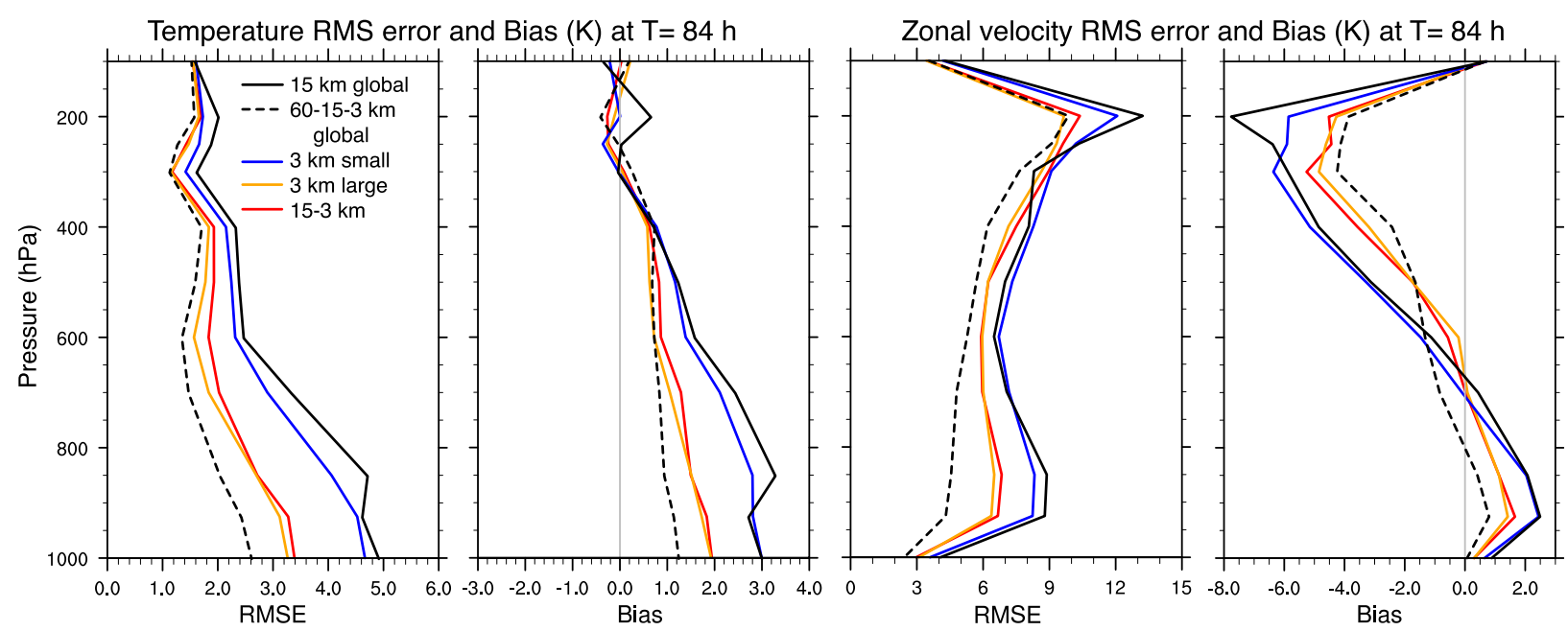

FIG. 12. RMSE and bias for temperature and zonal winds as a function of height, computed within the region covered by the small 3-km regional mesh (red circles in Fig. 4).

greatly reduces the mismatch for the inner nests. Multiple nesting can also be used in a one-way configuration, but this greatly increases the likelihood of mismatched solutions for the inner nest boundaries. A more aggressive approach is to use spectral nudging in a regional model to minimize the difference in the solutions at the larger scales (Hong and Kanamitsu 2014). Within MPAS, we advocate using a configuration where the mesh is coarsened as it approaches the boundary so that the relaxation zone is wider and anomalous convergence and divergence are reduced, as illustrated in the example in Fig. 14.

\section{Optimal regional model configurations}

\section{a. Error sources}

Optimal regional model configurations are based on the minimization of some error metric, and the three MPAS regional configurations, along with the global 60-15-3-km configuration, were used to attempt to understand various components of simulation error. We have removed model error relative to the actual atmosphere by using a perfect-model test configuration (i.e., by computing simulation error relative to a uniform high-resolution global simulation). The behavior of the error norms presented in section 3 is consistent with studies of error growth and predictability in atmospheric models (Judt 2018, and references therein). The error norms increase over time, reflecting the nonlinear behavior of the atmosphere and the expectation that the solution error norms will grow, with faster growth at smaller spatial scales, until the error saturates. The error norms in Figs. 10 and 11 do not indicate that the solution errors are saturated at the end of the simulation period (5 days), suggesting that there is still some forecast skill in the larger scales relative to the perfect-model $3-\mathrm{km}$ global solution in these simulations. Because we have removed errors associated with the model's ability to simulate the actual atmosphere by using the perfectmodel approach, these behaviors of the error norms should be characterized as providing a lower bound on solution errors in actual forecast applications (i.e., errors relative to the observed atmosphere).

There are at least four remaining sources of error in the MPAS regional configurations tested in this study:

1) Errors arising from the formulation of the lateral boundary conditions, including the filtering applied in the relaxation region.

2) Errors in the driving solution, including temporal sampling errors.

3) Model errors in the interior coarse-mesh region in stretched mesh regional configurations relative to uniform high-resolution regional configurations, including errors arising from terrain mismatches and errors arising purely from the resolution differences.

4) Errors associated with the behavior of model physics on different mesh resolutions.

All these errors are present in the simulations but are not easily distinguished. The results show that small regional-domain simulation errors are dominated by those of the driving simulation in most error measures, illustrating the impact of downscaling; the $3-\mathrm{km}$ small region simulation errors are similar to those of the $15-\mathrm{km}$ driving solution, except for the additional smallscale structure. The use of stretched meshes to allow for a large domain, or using a large fine mesh, reduces 

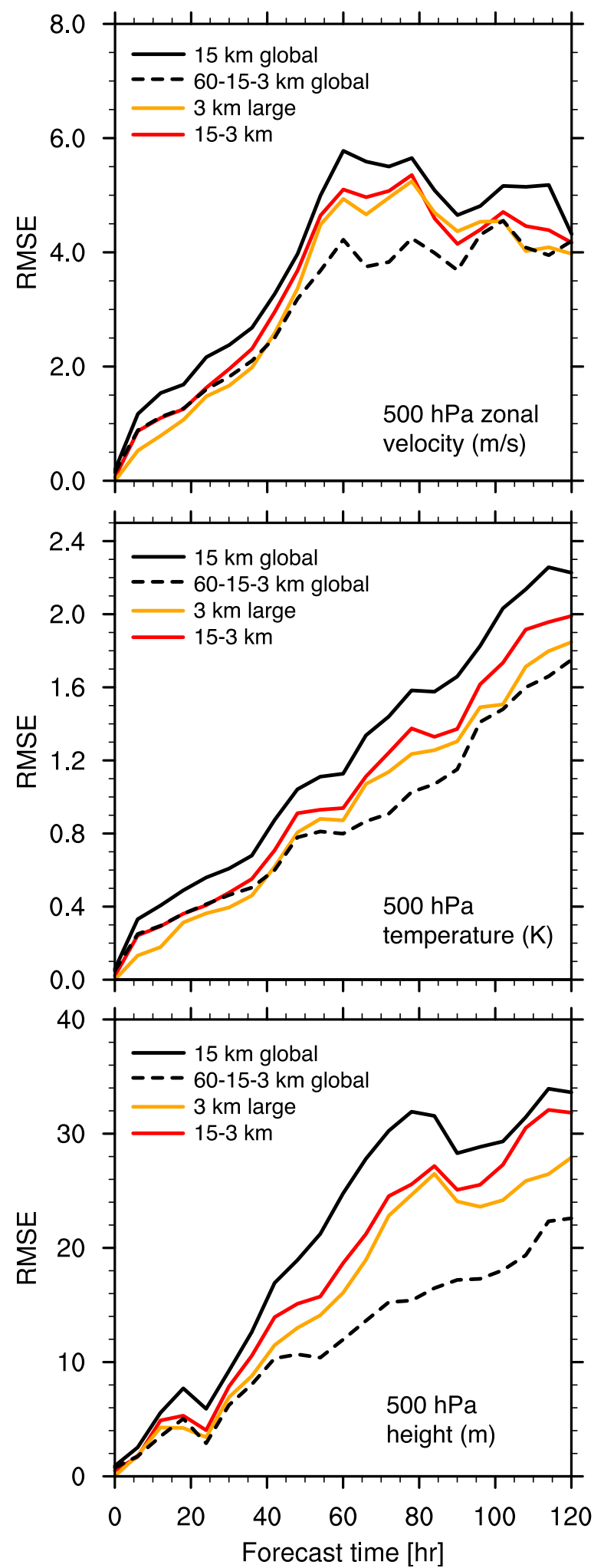

FIG. 13. As in Fig. 10, but for errors within the region covered by the large regional mesh (blue circle in Fig. 4). simulation errors. The stretched mesh is beneficial because it allows for a larger boundary zone at reduced cost relative to a large high-resolution configuration, similar to that found by anisotropic stretching of regional rectangular grids by Davies (2017). The gradual stretching of the unstructured mesh also filters finescale structures before they reach the boundary zone, reducing stress on the relaxation region formulation and filtering. As noted in the previous section, the variablemesh configurations are more robust because they reduce the solution mismatch problems, compared to a large uniform-mesh configuration. There is additional error introduced by stretching the mesh, as opposed to using a large high-resolution uniform mesh as shown in all quantitative error measures (i.e., Figs. 10-13), although the additional error is only noticed at later simulation times in most quantitative measures. The 60-15-3-km global simulation is much more accurate than the $15-\mathrm{km}$ global simulation used to drive the regional simulations. This fact suggests that much of the error in the driving solution (the $15-\mathrm{km}$ global simulation) has its origin in the lack of high resolution over the central United States, and this holds through 5 days, even though one might expect the increased error from the $60-\mathrm{km}$ portion of the $60-15-3-\mathrm{km}$ simulation to increase the regional error metrics toward the end of the simulation period.

\section{b. Simulation cost and efficiency}

Table 1 presents the costs of the 5-day simulations for the regional and global MPAS model mesh configurations. The costs are given relative to the cost of the $3-\mathrm{km}$ small region simulation, and they represent the relative approximate number of processor hours needed to produce the simulations. The estimate is based on the assumption that the MPAS model scales perfectly. The domain entries are ordered by a qualitative assessment of the overall solution error, beginning with the reference solution.

We define efficiency as solution accuracy relative to simulation cost; thus, optimally efficient configurations maximize accuracy for a given cost or minimize cost for a given accuracy. A user must define what measure or measures of accuracy are to be used to determine efficiency and how to weight the accuracy measures to determine if increased computational costs justify a given error reduction. Herein, we consider qualitatively the cost and accuracy of the configurations we have tested. Our test results suggest the following:

1) If a regional configuration is employed primarily for downscaling so as to explicitly simulate small-scale structure (e.g., convection) over a short time period 

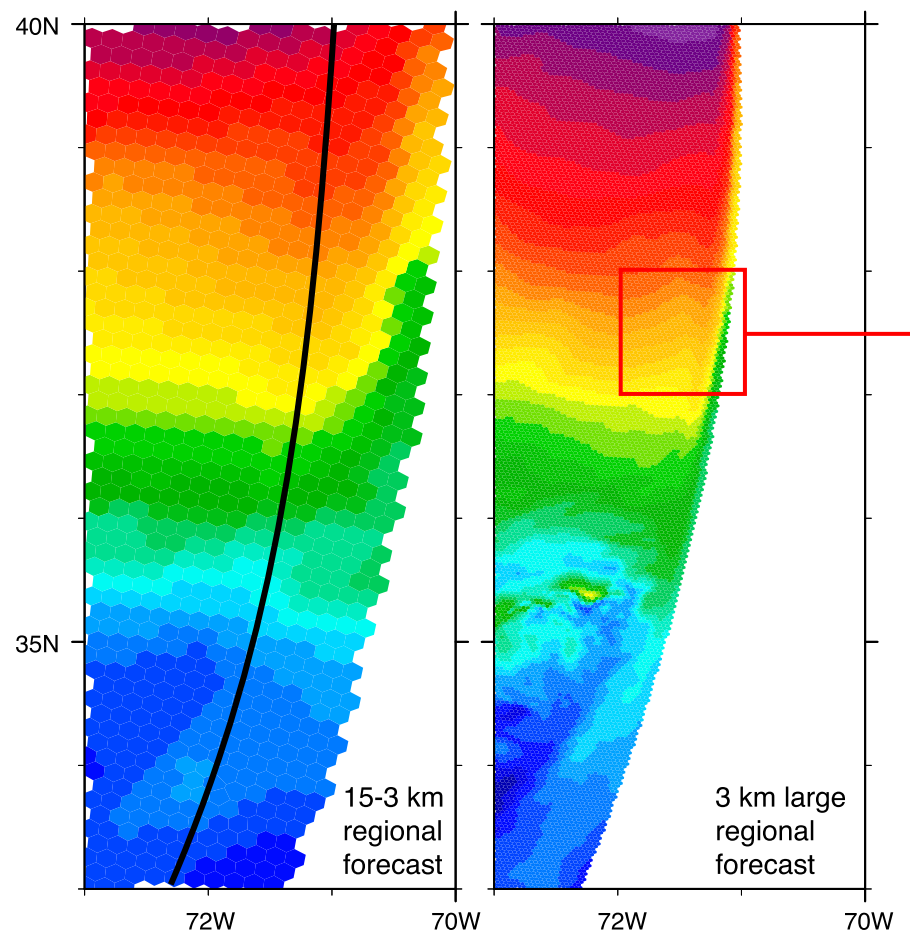

\section{$500 \mathrm{hPa}$ zonal windspeed forecast hour 84}

FIG. 14. Contour plots of the 500-hPa zonal wind at simulation hour 84 in the boundary regions of the 3-km large-region simulation and the 15-3-km regional simulation. The black lines in the leftmost and rightmost panels indicate the edge of the boundary zone. Ridges in the zonal wind contours at the edge of the boundary zone are visible in both solutions.

(a few days), then using a small domain and uniform resolution makes sense as long as boundary mismatch problems are not an issue. It is the least expensive simulation here.

2) If a regional domain over which downscaling is desired is large enough that boundary solution mismatches are an issue, then extending the domain using a variable-resolution mesh can be economical and may increase accuracy further out in time.

3) Extending a fine-resolution region to a larger domain to decrease errors is much more expensive relative to using larger stretched regional meshes or global stretched meshes, but it does result in somewhat lower solution error in some metrics at later simulation time relative to a stretched mesh configuration. While it extends the high-resolution downscaling to a larger region, it is also more prone to solution mismatch issues at the boundaries. In the test case presented here, the LBC errors are smaller than the model (resolution related) errors; thus, this configuration performs the best.

4) It may be more efficient to run a global stretched mesh, compared to a regional mesh, if the global uniform mesh driving solution is considered in the overall cost-the cost of the $15-3-\mathrm{km}$ regional solution plus the $15-\mathrm{km}$ global solution needed to drive it
(4.19) is larger than the $60-15-3-\mathrm{km}$ global simulation cost (3.70). The global 60-15-3-km simulation has the lowest errors of all the simulations by the end of day 2, and the superiority of this simulation, compared to the other regional simulations, persists through day 5 in most measures over the entire troposphere.

Overall, as the desired downscaling region is enlarged and the time period of the simulation is lengthened, the use of an MPAS stretched regional mesh becomes more efficient relative to a uniform high-resolution regional mesh, and, depending on the accuracy metrics, a global stretched mesh may be the most efficient.

\section{Summary}

Existing regional atmospheric model boundary treatments, widely used in rectangular mesh models, have been adapted to the MPAS atmospheric model and its centroidal Voronoi mesh and discretizations. Using a perfectmodel testing framework with a global 3-km simulation serving as truth, the regional configurations of MPAS produce results that show the benefits of downscaling similar to those produced by other regional atmospheric models [e.g., realistic and accurate finer-scale structure (explicit convection)] and reduced errors relative to the 
driving solution in most error measures over most of the 5-day simulation.

Consistent with results from many other studies, we have found that enlarging a regional domain will generally reduce simulation error. The general centroidal Voronoi meshes used by MPAS allow for smoothly varying resolution, and when the variable-resolution capability is used to extend the lateral boundaries away from the downscaling region, most of the error reduction is recovered compared with a uniform highresolution mesh configuration over the same extended region, and at a much-reduced cost.

As is the case for other regional models, spurious boundary artifacts arising from solution mismatches between the regional model and the driving solution are also found in the MPAS-A regional results-in this case, with a large $(5: 1)$ cell spacing ratio at the boundary. The variable-resolution MPAS regional mesh allows for a larger and less expensive relaxation zone, helping to ameliorate the negative effects of these differences near the lateral boundary that evolve between the model solution and the driving solution. The larger (wider) relaxation zone provided with a variable mesh results in more robust (stable) model configurations. Additionally, having the resolution coarsen as the boundary zone is approached results in filtering of scales not represented in the driving solution, along with a reduction of the filtering needed within the lateral boundary region itself. We believe that it is optimal and most efficient to match the regional model boundary-zone resolution to the driving model resolution because in this way, we are making use of all the resolved structure in the driving solution for the minimum cost and making use of the mesh-aware filtering of the variable-resolution mesh solver numerics.

For applications, optimal configurations depend on forecast error metrics and required accuracy measures, desired simulation region and length, and the available resources for forecast generation. For regional modeling applications focused on downscaling to convectionpermitting scales, the limited results in this study suggest that regional models can be effective for forecast lengths of a few days, with larger domain configurations maintaining that effectiveness for a longer period than smaller domains. Depending on how accuracy and cost are weighted, using a variable-resolution mesh to expand the regional domain and decrease solution error may be more cost effective than using a large uniformresolution configuration. For forecast periods out past a few days, however, the benefits of using a global variable-resolution mesh become apparent, especially compared to the high cost of using a large regional highresolution mesh.
Acknowledgments. We would like to acknowledge support for this work from the Korean Institute of Science and Technology Information under Grants C16016 and MMM17313. Computing support for this work was provided by NCAR's Computational and Information Systems Laboratory. We would also like to acknowledge the three anonymous reviewers of this paper for helping to improve the manuscript. The National Center for Atmospheric Research is supported by the National Science Foundation.

\section{REFERENCES}

Bonaventura, L., A. Iske, and E. Miglio, 2011: Kernel-based vector field reconstruction in computational fluid dynamic models. Int. J. Numer. Methods Fluids, 66, 714-729, https://doi.org/ 10.1002/fld.2279.

Castro, C. L., R. A. Pielke, and G. Leoncini, 2005: Dynamical downscaling: Assessment of value retained and added using the Regional Atmospheric Modeling System (RAMS). J. Geophys. Res., 110, D05108, https://doi.org/10.1029/2004JD004721.

Côté, J., J.-G. Desmarais, S. Gravel, A. Méthot, A. Patoine, M. Roch, and A. Staniforth, 1998: The Operational CMCMRB Global Environmental Multiscale (GEM) Model. Part II: Results. Mon. Wea. Rev., 126, 1397-1418, https://doi.org/ 10.1175/1520-0493(1998)126<1397:TOCMGE > 2.0.CO;2.

Davies, H., and R. E. Turner, 1977: Updating prediction models by dynamical relaxation: An examination of the technique. Quart. J. Roy. Meteor. Soc., 103, 225-245, https://doi.org/10.1002/ qj.49710343602.

Davies, T., 2014: Lateral boundary conditions for limited area models. Quart. J. Roy. Meteor. Soc., 140, 185-196, https://doi.org/ 10.1002/qj.2127.

— 2017: Dynamical downscaling and variable resolution in limited-area models. Quart. J. Roy. Meteor. Soc., 143, 209-222, https://doi.org/10.1002/qj.2913.

de Elía, R., R. Laprise, and B. Denis, 2002: Forecasting skill limits of nested, limited-area models: A perfect-model approach. Mon. Wea. Rev., 130, 2006-2023, https://doi.org/10.1175/15200493(2002)130<2006:FSLONL>2.0.CO;2.

Done, J., C. A. Davis, and M. Weisman, 2004: The next generation of NWP: Explicit forecasts of convection using the Weather Research and Forecasting (WRF) Model. Atmos. Sci. Lett., 5, 110-117, https://doi.org/10.1002/asl.72.

Fowler, L. D., W. C. Skamarock, G. A. Grell, S. R. Freitas, and M. Duda, 2016: Analyzing the Grell-Freitas convection scheme from hydrostatic to nonhydrostatic scales within a global model. Mon. Wea. Rev., 144, 2285-2306, https://doi.org/ 10.1175/MWR-D-15-0311.1.

Grell, G. A., and S. R. Freitas, 2014: A scale and aerosol aware stochastic convective parameterization for weather and air quality modeling. Atmos. Chem. Phys., 14, 5233-5250, https:// doi.org/10.5194/acp-14-5233-2014.

Hodur, R., 1997: The Naval Research Laboratory's Coupled Ocean/ Atmosphere Mesoscale Prediction System (COAMPS). Mon. Wea. Rev., 125, 1414-1430, https://doi.org/10.1175/15200493(1997)125<1414:TNRLSC > 2.0.CO;2.

Hong, S.-Y., and M. Kanamitsu, 2014: Dynamical downscaling: Fundamental issues from an NWP point of view and recommendations. Asia-Pac. J. Atmos. Sci., 50, 83-104, https://doi.org/ 10.1007/s13143-014-0029-2. 
Judt, F., 2018: Insights into atmospheric predictability through global convection-permitting model simulations. J. Atmos. Sci., 75, 1477-1497, https://doi.org/10.1175/JAS-D-17-0343.1.

Klemp, J. B., 2011: A terrain-following coordinate with smoothed coordinate surfaces. Mon. Wea. Rev., 139, 2163-2169, https:// doi.org/10.1175/MWR-D-10-05046.1.

- W. C. Skamarock, and J. Dudhia, 2007: Conservative splitexplicit time integration methods for the compressible nonhydrostatic equations. Mon. Wea. Rev., 135, 2897-2913, https://doi.org/10.1175/MWR3440.1.

Oliger, J., and A. Sundström, 1978: Theoretical and practical aspects of some initial boundary value problems in fluid dynamics. SIAM J. Appl. Math., 35, 419-446, https://doi.org/10.1137/0135035.

Park, S.-H., J. B. Klemp, and W. C. Skamarock, 2014: A comparison of mesh refinement in the global MPAS-A and WRF Models using an idealized normal-mode baroclinic wave simulation. Mon. Wea. Rev., 142, 3614-3634, https://doi.org/10.1175/MWR-D-14-00004.1.

Ringler, T., J. Thuburn, J. B. Klemp, and W. C. Skamarock, 2010: A unified approach to energy conservation and potential vorticity dynamics for arbitrarily-structured C-grids. J. Comput. Phys., 229, 3065-3090, https://doi.org/10.1016/j.jcp.2009.12.007.

, D. Jacobsen, L. Ju, M. Gunzburger, M. Duda, and W. C. Skamarock, 2011: Exploring a multiresolution modeling approach within the shallow-water equations. Mon. Wea. Rev., 139, 3348 3368, https://doi.org/10.1175/MWR-D-10-05049.1.

Skamarock, W. C., and A. Gassmann, 2011: Conservative transport schemes for spherical geodesic grids: High-order flux operators for ODE-based time integration. Mon. Wea. Rev., 139, 2962-2975, https://doi.org/10.1175/MWR-D-10-05056.1.

— , and Coauthors, 2008: A description of the Advanced Research WRF version 3. NCAR Tech. Note NCAR/TN475+STR, 113 pp., https://doi.org/10.5065/D68S4MVH.

— J. B. Klemp, M. G. Duda, L. D. Fowler, S.-H. Park, and T. Ringler, 2012: A multiscale nonhydrostatic atmospheric model using centroidal Voronoi tesselations and C-grid staggering. Mon. Wea. Rev., 140, 3090-3105, https://doi.org/ 10.1175/MWR-D-11-00215.1.

Wicker, L. J., and W. C. Skamarock, 2002: Time-splitting methods for elastic models using forward time schemes. Mon. Wea. Rev., 130, 2088-2097, https://doi.org/10.1175/1520-0493(2002)130<2088: TSMFEM >2.0.CO;2.

Xue, M., K. K. Droegemeier, and V. Wong, 2000: The Advanced Regional Prediction System (ARPS) -A multi-scale nonhydrostatic atmospheric simulation and prediction model. Part I: Model dynamics and verification. Meteor. Atmos. Phys., 75, 161-193, https://doi.org/10.1007/s007030070003. 